\title{
COMPACTNESS FOR CONFORMAL METRICS WITH CONSTANT $Q$ CURVATURE ON LOCALLY CONFORMALLY FLAT MANIFOLDS
}

\author{
Jie QING AND DAVID RASKe
}

\begin{abstract}
In this note we study the conformal metrics of constant $Q$ curvature on closed locally conformally flat manifolds. We prove that for a closed locally conformally flat manifold of dimension $n \geq 5$ and with Poincarë exponent less than $\frac{n-4}{2}$, the set of conformal metrics of positive constant $Q$ and positive scalar curvature is compact in the $C^{\infty}$ topology.
\end{abstract}

\section{Introduction}

Let $(M, g)$ be a compact manifold without boundary of dimension higher than 4. Let

$$
Q[g]=-\frac{n-4}{4(n-1)} \Delta R+\frac{(n-4)\left(n^{3}-4 n^{2}+16 n-16\right)}{16(n-1)^{2}(n-2)^{2}} R^{2}-\frac{2(n-4)}{(n-2)^{2}}|R i c|^{2}
$$

be the so-called $Q$-curvature, where $R$ is the scalar curvature, Ric is the Ricci curvature. And let

$$
P[g]=(-\Delta)^{2}-\operatorname{div}_{g}\left(\left(\frac{(n-2)^{2}+4}{2(n-1)(n-2)} R g-\frac{4}{n-2} R i c_{g}\right) d\right)+Q[g]
$$

be the so-called Paneitz-Branson operator. It is known that

$$
P[g] w=Q\left[g_{w}\right] w^{\frac{n+4}{n-4}}
$$

which is called the Paneitz-Branson equation, where $g_{w}=w^{\frac{4}{n-4}} g(c f . \quad[\mathrm{P}][\mathrm{Br}]$ [DHL] [DMA] ). We consider the equation (1.3) as a fourth order analogue of the well-known scalar curvature equation

$$
L[g] u=R\left[g_{u}\right] u^{\frac{n+2}{n-2}},
$$


where

$$
L[g]=-\frac{4(n-1)}{n-2} \Delta+R
$$

is the so-called conformal Laplacian and $g_{u}=u^{\frac{4}{n-2}} g$. The well-known Yamabe problem in conformal geometry is to find a metric, in a given class of conformal metrics, which is of constant scalar curvature, i.e. to solve

$$
L[g] u=Y u^{\frac{n+2}{n-2}}
$$

on a given manifold $(M, g)$ for some positive function $u$ and a constant $Y$. The affirmative resolution to the Yamabe problem was given in [Sc1] after other notable works [Ya] [Tr] $[\mathrm{Au}]$.

Recently, there are more and more interests in using higher order partial differential equations in the study of conformal geometry, particularly after some successes in dimension 4 in $[\mathrm{CY}]$ (references therein). One major hurdle that stands in the way to this higher order approach is the lack of the maximum principles for the higher order partial differential equations like (1.3). One, for example, would like to say that a nonnegative solution $w$ to (1.3) is necessarily positive, and therefore gives rise to a metric $g_{w}=w^{\frac{4}{n-4}} g$.

In this note we consider closed locally conformally flat manifolds with positive Yamabe constant of dimension higher than 4. By work in [SY], we know that such manifolds are all Kleinian in the sense that, there is a Kleinian group $\Gamma$ such that $(M,[g])$ is conformally equivalent to $\Omega(\Gamma) / \Gamma$, where $\Omega(\Gamma)$ is set of ordinary points of the Kleinian group $\Gamma$ on $S^{n}$. The developing map is a conformal diffeomorphism from the universal cover $\tilde{M}$ of $M$ to $\Omega(\Gamma) \subset S^{n}$. The Kleinian group $\Gamma$ is the so-called holonomy representation of the fundamental group of $M$. Hence we may turn to study solutions to

$$
(-\Delta)^{2} \hat{w}=Q\left(\hat{g}_{w}\right) \hat{w}^{\frac{n+4}{n-4}}
$$

on a domain $\hat{\Omega} \subset R^{n}$ instead of solutions to the Paneitz-Branson equation (1.3) on $M$. Given a Kleinian group $\Gamma$, the so-called Poincaré exponent is defined as:

$$
\delta(\Gamma)=\inf \left\{\delta>0: \sum_{\gamma \in \Gamma}\left|\gamma^{\prime}(x)\right|^{\delta}<\infty, \forall x \in S^{n}\right\}
$$

Inspired by the work of Wei and $\mathrm{Xu}$ in $[\mathrm{WX}]$, where they managed to use some maximum principle and the moving plane method to classify all positive solutions to (1.6) on entire $R^{n}$ with $Q\left(\hat{g}_{w}\right)$ a positive constant, we have 
Theorem 1.1. Suppose that $\left(M^{n}, g\right)$ is a closed locally conformally flat manifold with positive Yamabe constant and its Poincaré exponent less than $\frac{n-4}{2}$. Then any nontrivial nonnegative solution to

$$
P[g] u=Q u^{\frac{n+4}{n-4}}
$$

on $M$ with positive constant $Q$ has to be strictly positive on $M$.

The assumption on Poincaré exponent gives us the integrability condition to use the idea from [WX]. We also adopt the nice and geometric use of the moving plane method of Schoen from [Sc2] and prove a local convexity result analogous to the one in [Sc2]. One key point is to use the Hopf maximum principle for elliptic systems instead (cf. [Q]).

Lemma 1.2. Let $(\Omega, \tilde{g})$ be the universal cover of a closed manifold $\left(M^{n}, g\right)$ where $n>4, \Omega$ is not $S^{n}, \tilde{g}=v^{\frac{4}{n-4}} g_{1}$ and $g_{1}$ is the standard round metric on $S^{n}$. And suppose that $Q[\tilde{g}]$ is a positive constant and that the scalar curvature $R[\tilde{g}]$ is positive. Then any small round ball $B \subset \Omega \subset S^{n}$ is geodesically convex with respect to the metric $\tilde{g}$.

Combining Lemma 1.2 in the above with the classification given in [WX], we therefore derive our $L^{\infty}$ estimate:

Theorem 1.3. Let $(M, g)$ be a closed locally conformally flat manifold of dimension greater than 4 with positive Yamabe constant. And suppose that $(M, g)$ is not conformal to $\left(S^{n}, g_{1}\right)$. Then there exists $C>0$ such that, if $g_{u}=u^{\frac{4}{n-4}} g$ is of positive scalar curvature and constant $Q$-curvature 1 , then

$$
\|u\|_{L^{\infty}(M)} \leq C
$$

Similar to the study of Yamabe problem, we consider

$$
P(M,[g])=\inf _{u^{\frac{4}{n-4}} g \in[g]} \frac{\int_{M}(Q d v)\left[u^{\frac{4}{n-4}} g\right]}{\left(\int_{M} d v\left[u^{\frac{4}{n-4}} g\right]\right)^{\frac{n-4}{n}}} .
$$

We will refer to $P(M,[g])$ as the Paneitz constant in this paper. Clearly the Paneitz constant $P(M,[g])$ is a conformal invariant. As a consequence of Theorem 1.1 and Theorem 1.3 in the above, here is our main theorem:

Theorem 1.4. Let $\left(M^{n}, g\right)$ be a closed, locally conformally flat manifold of dimension $n$ greater than 4 with positive Yamabe constant and Poincaré exponent less than $\frac{n-4}{2}$. Suppose that $(M, g)$ is not conformal to $\left(S^{n}, g_{1}\right)$ and that the Paneitz 
constant $P(M,[g])$ is positive. Then the set of metrics conformal to $g$ on $M$ with positive scalar curvature and constant $Q$-curvature 1 is compact in $C^{\infty}$ topology. That is, there exists $C_{k}>0$ for each $k \in N$ such that, if $g_{u}=u^{\frac{4}{n-4}} g$ is of positive scalar curvature and constant $Q$-curvature 1 , then

$$
\|u\|_{C^{k}(M)}+\left\|\frac{1}{u}\right\|_{C^{k}(M)} \leq C_{k}
$$

We would like to mention that it was proven in $[\mathrm{CHY}][\mathrm{G}]$ that the Hausdorff dimension of the limit set of the Kleinian group associated with a closed, locally conformally flat manifold $(M, g)$ of dimension higher than 4 with positive scalar curvature and positive $\sigma_{2}$-curvature is less than $\frac{n-4}{2}$, which in turn implies the Poincaré exponent is less than $\frac{n-4}{2} . \sigma_{2}$-curvature is the second symmetric function of the eigenvalues of Weyl-Schouten tensor $R_{i j}-\frac{1}{2(n-1)} R g_{i j}$. It is certainly conceivable that the positivity of both the Paneitz constant and Yamabe constant should imply the Poincaré exponent is less than $\frac{n-4}{2}$.

Finally we like to mention that after submission of this paper we learnt the announcement of Hebey and Robert [HR], where they had obtained some results similar to the ones in this paper. One difference is that our compactness is for the metrics, while their compactness is for the nonnegative solutions. In other words, we have Theorem 1.1 and the positive lower bound in this paper. Our approach is to use the moving plane method to obtain the geodesic convexity of round balls and exclude the possibility of blow-ups, while the analysis in [HR] is based on the study of the asymptotic analytic behavior of solutions near the blow-up points and uses the strong positivity assumption on the Green function.

The organization of the note is as follows: in Section 2 we will recall more about Kleinian groups and works in $[\mathrm{SY}]$ to prove Theorem 1.1. In Section 3 we will adopt the moving plane approach from [Sc2] to prove Lemma 1.2. Finally in Section 4 we use the blow-up method to obtain $L^{\infty}$ estimates and prove our main theorem.

Acknowledgement We would like to thank Professor Alice Chang and Professor Paul Yang for their interests in this work.

\section{Positivity of solutions to Paneitz-Branson equations}

Suppose that $\left(M^{n}, g\right)$ is a closed locally conformally flat manifold with positive Yamabe constant. Then by works in $[\mathrm{SY}]$ we know that the developing map from the universal cover $(\tilde{M}, \tilde{g})$ into $\left(S^{n}, g_{1}\right)$ is a conformal embedding, where $g_{1}$ is the standard round metric on $S^{n}$. And the deck transformation group of the universal covering becomes a discrete subgroup of the group of conformal transformation of $S^{n}$, which is called a Kleinian group. This Kleinian group $\Gamma$ is said to be the 
holonomy representation of the fundamental group of $M$. The image of the developing map from the universal cover $\tilde{M}$ is the set $\Omega(\Gamma)$ of ordinary points for the Kleinian group $\Gamma$, and $(M,[g])$ is conformally equivalent to $\Omega(\Gamma) / \Gamma$, which is called the Kleinian manifold associated with the group $\Gamma$. Therefore we may consider $(\Omega(\Gamma), \tilde{g})$ as the universal cover for $(M, g)$ and $\tilde{g}=\tilde{\eta}^{\frac{4}{n-4}} g_{1}$. Hence

$$
P\left[g_{1}\right] \tilde{\eta}=Q[\tilde{g}] \tilde{\eta}^{\frac{n+4}{n-4}} .
$$

Using stereographic projection

$$
\psi: \hat{\Omega} \subset R^{n} \longrightarrow \Omega(\Gamma) \subset S^{n}
$$

with respect to some point in $\Omega(\Gamma) \subset S^{n}$, we may write $\hat{g}=\psi^{*} \tilde{g}=\hat{\eta}^{\frac{4}{n-4}} g_{0}$, where $g_{0}$ is the Euclidean metric and $\hat{\eta}=(\tilde{\eta} \circ \psi)\left(\frac{2}{1+|x|^{2}}\right)^{\frac{n-4}{2}}$, and

$$
P\left[g_{0}\right] \hat{\eta}=Q[\hat{g}] \hat{\eta}^{\frac{n+4}{n-4}}
$$

Notice that $P\left[g_{0}\right]=(-\Delta)^{2}$. Suppose that $g_{u}=u^{\frac{4}{n-4}} g$ is another metric in $[g]$ on $M$ and $\phi: \Omega(\Gamma) \rightarrow M$ is the covering map, which is the composition of the developing map and the universal covering. Then

$$
\hat{g}_{u}=\hat{u}^{\frac{4}{n-4}} g_{0}
$$

where $\hat{u}=(u \circ \phi \circ \psi) \hat{\eta}$. Then

$$
P[g] u=Q\left[g_{u}\right] u^{\frac{n+4}{n-4}} \quad \text { on } M
$$

and

$$
(-\Delta)^{2} \hat{u}=Q\left[\hat{g}_{u}\right] \hat{u}^{\frac{n+4}{n-4}} \quad \text { in } \hat{\Omega} \subset R^{n}
$$

where $Q\left[\hat{g}_{u}\right]=Q\left[g_{u}\right] \circ \phi \circ \psi$. Due to the invariant property of the above equations, one may easily see that, for a nonnegative function $v$ satisfying

$$
P[g] v=Q v^{\frac{n+4}{n-4}} \quad \text { on } M
$$

for some function $Q$ on $M, \hat{v}=(v \circ \phi \circ \psi) \hat{\eta}$ satisfies

$$
(-\Delta)^{2} \hat{v}=\hat{Q} \hat{v}^{\frac{n+4}{n-4}} \quad \text { in } \hat{\Omega} \subset R^{n}
$$


for the function $\hat{Q}=Q \circ \phi \circ \psi$ on $\hat{\Omega}$.

One very important quantity of a Kleinian group $\Gamma$ is the so-called Poincaré exponent

$$
\delta(\Gamma)=\inf \left\{\delta>0: \sum_{\gamma \in \Gamma}\left|\gamma^{\prime}(x)\right|^{\delta}<\infty, \forall x \in S^{n}\right\} .
$$

Another very important quantity is the Hausdorff dimension $d(\Gamma)$ of the limit set $L(\Gamma)$. Due to a theorem of Patterson and Sullivan one knows that $\delta(\Gamma)=d(\Gamma)$ when $\Gamma$ is geometrically finite. In our cases, the Kleinian group associated with a closed, locally conformally flat manifold of positive Yamabe constant is always geometrically finite (cf. [CQY2]). Now we are ready to state and prove an interesting property of a Kleinian manifold.

Lemma 2.1. Suppose that $(M,[g])$ is a Kleinian manifold $\Omega(\Gamma) / \Gamma$ with $\delta(\Gamma)<$ $\frac{n-4}{2}$. And suppose that $v$ is a nonnegative function on $M$ and $\tilde{v}=(v \circ \phi) \tilde{\eta}$. Then

$$
\int_{S^{n}} \tilde{v}^{\frac{n+4}{n-4}} d v_{g_{1}}<\infty
$$

Proof. We need only to show that

$$
\int_{\Omega(\Gamma)} \tilde{v}^{\frac{n+4}{n-4}} d v_{g_{1}}<\infty
$$

Because the Hausdorff dimension $d(\Gamma)$ of $S^{n} \backslash \Omega(\Gamma)$ is less than $\frac{n-4}{2}$ by the theorem of Patterson and Sullivan. Notice that, if we let $F$ be a fundamental domain of $\Gamma$,

$$
\int_{\Omega(\Gamma)} \tilde{v}^{\frac{n+4}{n-4}} d v_{g_{1}}=\sum_{\gamma \in \Gamma} \int_{\gamma(F)} \tilde{v}^{\frac{n+4}{n-4}} d v_{g_{1}}
$$

Since $\gamma$ is an isometry for $g$, we have

$$
\tilde{\eta}^{\frac{4}{n-4}}(x) g_{1}=\gamma^{*}\left(\tilde{\eta}^{\frac{4}{n-4}}(x) g_{1}\right)=\tilde{\eta}^{\frac{4}{n-4}}(\gamma(x))\left|\gamma^{\prime}\right|^{2} g_{1}
$$

and

$$
\tilde{v}(\gamma(x))=v \circ \phi(\gamma(x)) \tilde{\eta}(\gamma(x))=\tilde{v}(x)\left|\gamma^{\prime}(x)\right|^{-\frac{n-4}{2}} .
$$

Hence

$$
\int_{\gamma(F)} \tilde{v}^{\frac{n+4}{n-4}} d v_{g_{1}}=\int_{F} \tilde{v}(x)^{\frac{n+4}{n-4}}\left|\gamma^{\prime}(x)\right|^{-\frac{n+4}{2}}\left|\gamma^{\prime}(x)\right|^{n} d v_{g_{1}}=\int_{F} \tilde{v}(x)^{\frac{n+4}{n-4}}\left|\gamma^{\prime}(x)\right|^{\frac{n-4}{2}} d v_{g_{1}} .
$$


Therefore

$$
\int_{\Omega(\Gamma)} \tilde{v}^{\frac{n+4}{n-4}} d v_{g_{1}}=\int_{F}\left(\sum_{\gamma \in \Gamma}\left|\gamma^{\prime}(x)\right|^{\frac{n-4}{2}}\right) \tilde{v}(x)^{\frac{n+4}{n-4}} d v_{g_{1}}
$$

which is finite when the Poincaré exponent, $\delta(\Gamma)<\frac{n-4}{2}$. Thus the proof is completed.

Corollary 2.2. Suppose that $(M,[g])$ is a closed Kleinian manifold $\Omega(\Gamma) / \Gamma$ with $\delta(\Gamma)<\frac{n-4}{2}$. And suppose that $v$ is a nonnegative function on $M$ and $\hat{v}=(v \circ \phi \circ \psi) \hat{\eta}$. Then

$$
\int_{U} \hat{v}^{\frac{n+4}{n-4}} d v_{g_{0}}<\infty
$$

for any bounded domain $U \subset R^{n}$.

Proof. Notice that

$$
\hat{v}=\tilde{v} \circ \psi\left(\frac{2}{1+|x|^{2}}\right)^{\frac{n-4}{2}} .
$$

Hence

$$
\int_{U} \hat{v}^{\frac{n+4}{n-4}} d v_{g_{0}}=\int_{\psi(U)} \tilde{v}^{\frac{n+4}{n-4}}\left(\frac{2}{1+|x|^{2}}\right)^{-\frac{n-4}{2}} d v_{g_{1}} .
$$

Therefore the corollary is proved.

Next we state and prove a theorem on the positivity of solutions to the PaneitzBranson equation on $M$.

Theorem 2.3. Suppose that $(M,[g])$ is a closed Kleinian manifold $\Omega(\Gamma) / \Gamma$ with $\delta(\Gamma)<\frac{n-4}{2}$. And suppose that $v$ is a smooth nonnegative function on $M$ satisfying

$$
P[g] v=Q v^{\frac{n+4}{n-4}} \quad \text { on } M
$$

for some positive constant $Q$. Then $v>0$ on $M$ if it is not identically zero.

Proof. Due to the homogeneity of the Paneitz-Branson equation, we may simply assume $Q=1$ in the following. According to the above discussion, we have

$$
(-\Delta)^{2} w=w^{\frac{n+4}{n-4}}
$$


on $\hat{\Omega} \subset R^{n}$, where $w=(v \circ \phi \circ \psi) \hat{\eta}$. Let us denote $-\Delta w=u$. If we can show that $u \geq 0$ on $\hat{\Omega}$, then we easily see that $v>0$ on $M$ by the strong maximum principle. We will adopt the idea from the paper of Wei and Xu [WX] for the following argument.

Assume otherwise that $u(0)=-\Delta w(0)<0($ assume $0 \in \hat{\Omega}$ or after translation if necessary). Notice that $\hat{\Omega}=R^{n} \backslash \psi^{-1}(L(\Gamma))$ where $\psi^{-1}(L(\Gamma))$ is a compact subset of $R^{n}$ since $\psi$ is a stereographic projection with respect to a point inside $\Omega(\Gamma)$. By the assumptions, $w$ is smooth away from $\psi^{-1}(L(\Gamma))$. Let

$$
\bar{f}(r)=\frac{1}{\left|S^{n-1}(1)\right|} \int_{S^{n-1}} f(r \sigma) d \sigma
$$

denote the spherical average of the function $f$. Then

$$
\left\{\begin{array}{l}
-\Delta \bar{u}=\overline{w^{\frac{n+4}{n-4}}} \\
-\Delta \bar{w}=\bar{u}
\end{array}\right.
$$

at least for very small $r$ and very large $r$. Let us solve the above equations as follows:

$$
\begin{aligned}
r^{n-1} \bar{u}^{\prime} & =-\int_{0}^{r} s^{n-1} \overline{w^{\frac{n+4}{n-4}}} d s \\
& =-\frac{1}{\left|S^{n-1}(1)\right|} \int_{0}^{r} \int_{S^{n-1}(1)} w^{\frac{n+4}{n-4}}(r \sigma) s^{n-1} d \sigma d s \\
& =-\frac{1}{\left|S^{n-1}(1)\right|} \int_{B_{r}(0)} w^{\frac{n+4}{n-4}} d x .
\end{aligned}
$$

Therefore, by above corollary on the integrability, we conclude that $\bar{u}$ is differentiable for all $r$, monotonically decreasing, and

$$
\begin{gathered}
\bar{u}(r)=u(0)-\int_{0}^{r} s^{-n+1} \int_{0}^{s} t^{n-1} \overline{w^{n+4}}(t) d t d s \\
r^{n-1} \bar{w}^{\prime}(r)=-\int_{0}^{r} s^{n-1} \bar{u}(s) d s
\end{gathered}
$$

and

$$
\bar{w}(r)=w(0)-\int_{0}^{r} s^{-n+1} \int_{0}^{s} t^{n-1} \bar{u}(t) d t d s .
$$


Plugging our assumption $u(0)<0$ into (2.24), we have

$$
\bar{w}(r) \geq \int_{0}^{r} s^{-n+1} \int_{0}^{s} t^{n-1} \bar{u}(t) d t d s \geq \frac{-u(0)}{2 n} r^{2} .
$$

For convenience we denote $q=\frac{n+4}{n-4}$. Applying Jenson's inequality

$$
\bar{w}^{q} \leq \overline{w^{q}}
$$

and $(2.27)$ in $(2.24)$, as in [WX], we have

$$
\begin{aligned}
\bar{u}(r) & \leq-\left(\frac{-u(0)}{2 n}\right)^{q} \int_{0}^{r} s^{-n+1} \int_{0}^{s} t^{n-1+2 q} d t d s \\
& =-\left(\frac{-u(0)}{2 n}\right)^{q} \frac{1}{n+2 q} \frac{1}{2 q+2} r^{2 q+2}
\end{aligned}
$$

and

$$
\begin{aligned}
\bar{w}(r) & \geq\left(\frac{-u(0)}{2 n}\right)^{q} \frac{1}{n+2 q} \frac{1}{2 q+2} \int_{0}^{r} s^{-n+1} \int_{0}^{t} t^{n-1} t^{2 q+2} d t \\
& =\left(\frac{-u(0)}{2 n}\right)^{q} \frac{1}{n+2 q} \frac{1}{2 q+2} \frac{1}{n+2+2 q} \frac{1}{2 q+4} r^{2 q+4} \\
& \geq\left(\frac{-u(0)}{2 n}\right)^{q} \frac{1}{(n+4+2 q)^{4}} r^{2 q+4} .
\end{aligned}
$$

Now the idea is to iterate the above steps over and over. Let

$$
\sigma_{k}=q \sigma_{k-1}+4, \sigma_{0}=2
$$

Then

$$
\begin{aligned}
\sigma_{k} & =q^{2} \sigma_{k-2}+4 q+4=q^{3} \sigma_{k-3}+4 q^{2}+4 q+4 \\
& =q^{k} \sigma_{0}+4 \frac{q^{k}-1}{q-1} \\
& =2 q^{k}+\frac{4}{q-1} q^{k}-\frac{4}{q-1} .
\end{aligned}
$$

We may calculate and obtain the following after $k$ iterations,

$$
\bar{w}(r) \geq\left(\frac{-u(0)}{2 n}\right)^{q^{k}} \prod_{j=1}^{k} \frac{1}{\left(n+\sigma_{j}\right)^{4 q^{k-j}}} r^{\sigma_{k}} .
$$


To finish the proof we calculate

$$
\prod_{j=1}^{k} \frac{1}{\left(2 \sigma_{j}\right)^{4 q^{k-j}}} \geq \frac{1}{\left(4\left(1+\frac{2}{q-1}\right)\right)^{\sum_{j=1}^{k} 4 q^{k-j}}} \frac{1}{q^{\sum_{j=1}^{k} 4 j q^{k-j}}}
$$

where

$$
\sum_{j=1}^{k} 4 q^{k-j}=4 \frac{q^{k}-1}{q-1}
$$

and

$$
\sum_{j=1}^{k} 4 j q^{k-j}=4 q^{k} \sum_{j=1}^{k} j q^{-j} \leq 4 q^{k} \int_{1}^{\infty} x q^{-x} d x \leq 8 q^{k-1}
$$

Thus

$$
\bar{w}(r) \geq c_{1}\left(c_{2} r\right)^{\sigma_{k}}
$$

and $\bar{w}(r)$ goes to $\infty$ when $k$ goes to $\infty$ for $r>c_{2}$, where $c_{1}$ and $c_{2}$ are numbers independent of $k$, which creates contradiction.

\section{Convexity}

In this section we use the moving plane method to prove a convexity result that is similar to what Schoen obtained in [Sc2]. Suppose that $(M, g)$ is a closed locally conformally flat manifold with positive Yamabe constant but not conformally equivalent to $\left(S^{n}, g_{1}\right)$. Suppose that the Kleinian group $\Gamma$ is the holonomy representation of the fundamental group of $M$. Let $\Omega(\Gamma)$ be the set of ordinary points. Then we may assume $(\Omega(\Gamma), \tilde{g})$ is a Riemannian universal cover for $(M, g)$. Let us further assume that the $Q$-curvature of $(M, g)$ is constant 1 . Then, if let $\tilde{g}=u^{\frac{4}{n-4}} g_{1}$, where $g_{1}$ the standard round metric on $S^{n}$, we have $u>0$ and

$$
P\left[g_{1}\right] u=u^{\frac{n+4}{n-4}} \quad \text { in } \Omega(\Gamma) \subset S^{n} .
$$

And in this case, we easily see that

$$
u(x) \rightarrow+\infty, \quad \text { as } x \rightarrow L(\Gamma)=S^{n} \backslash \Omega(\Gamma) .
$$

Because, as a Riemannian universal cover for $(M, g),\left(\Omega(\Gamma), u^{\frac{4}{n-4}} g_{1}\right)$ is complete. Using stereographic projection with respect to some point $p_{0} \in \Omega(\Gamma)$

$$
\psi: \hat{\Omega} \subset R^{n} \rightarrow \Omega(\Gamma) \subset S^{n}
$$


we may work in the coordinates so that

$$
\left(\hat{\Omega}, v^{\frac{4}{n-4}} g_{0}\right)=\left(\Omega(\Gamma) \backslash\left\{p_{0}\right\}, u^{\frac{4}{n-4}} g_{1}\right)
$$

where

$$
v=(u \circ \psi(x))\left(\frac{2}{1+|x|^{2}}\right)^{\frac{n-4}{2}}>0 .
$$

Hence

$$
(-\Delta)^{2} v=v^{\frac{n+4}{n-4}} \quad \text { in } \hat{\Omega} \subset R^{n},
$$

which is equivalent to the system

$$
\left\{\begin{aligned}
-\Delta w & =v^{\frac{n+4}{n-4}} \\
-\Delta v & =w
\end{aligned} \quad \text { in } \hat{\Omega} \subset R^{n},\right.
$$

We observe

Lemma 3.1. Suppose $\left(\Omega, u^{\frac{4}{n-4}} g_{1}\right)$ is the universal cover of $(M, g)$. Then

$$
v(x) \rightarrow+\infty, \quad \text { as } x \rightarrow \psi^{-1}(L(\Gamma))=R^{n} \backslash \hat{\Omega} .
$$

In addition suppose that the scalar curvature for $(M, g)$ is positive. Then

$$
-\Delta v(x) \rightarrow+\infty, \quad \text { as } x \rightarrow \psi^{-1}(L(\Gamma))=R^{n} \backslash \hat{\Omega} .
$$

Proof. (3.6) is a direct consequence of (3.2). To prove (3.7) we simply notice that

$$
-\Delta v^{\frac{n-2}{n-4}}=\hat{R} v^{\frac{n+2}{n-4}} \quad \text { in } \hat{\Omega} \subset R^{n}
$$

and

$$
-\Delta v^{\frac{n-2}{n-4}}=\frac{n-2}{n-4} v^{\frac{2}{n-4}}(-\Delta v)-\frac{n-2}{n-4} \frac{2}{n-4} v^{-\frac{n-6}{n-4}}|\nabla v|^{2}
$$

where $\hat{R}=R \circ \phi \circ \psi(x)$. Therefore

$$
-\Delta v=\frac{n-4}{n-2} \hat{R} v^{\frac{n}{n-4}}+\frac{2}{n-4} v^{-1}|\nabla v|^{2} \geq \frac{n-4}{n-2} R v^{\frac{n}{n-4}} .
$$

Thus (3.7) holds.

Now we are ready to state and prove a convexity result. 
Theorem 3.2. Suppose that $\left(M^{n}, g\right)$ is a closed locally conformally flat manifold of dimension higher than 4 which is not conformally equivalent to $\left(S^{n}, g_{1}\right)$. And suppose that the scalar curvature of $(M, g)$ is positive and the $Q$-curvature of $(M, g)$ is constant 1 . Let $(\Omega(\Gamma), \tilde{g})$ be the Riemannian universal cover for $(M, g)$ associated with the developing map. Then any round ball $B \subset \Omega(\Gamma) \subset S^{n}$ is geodesically convex with respect to the metric $\tilde{g}$.

Proof. Let $B \subset \Omega(\Gamma)$ be any round ball on $S^{n}$. Let $q \in \partial B$ be any given point on the sphere. Notice that the surface $\partial B$ is umbilical with respect to the round metric as well as to the metric $\tilde{g}$ since $\tilde{g}$ is conformal to $g_{1}$. We use stereographic projection $\psi$ with respect to $q$ to construct a coordinate system in which $\partial B$ is the hyperplane at the origin, i.e. $\left\{x=\left(x_{1}, x_{2}, \cdot, x_{n}\right) \in R^{n}: x_{n}=0\right\}$, and the $x_{n}$-axis is in the direction of inward normal direction. Notice that we have that $\psi^{-1}(L(\Gamma))$ is located $R^{n}$ with $x_{n}<0$. Hence to prove convexity of $B$ with respect to the metric $\tilde{g}=v^{\frac{4}{n-4}} g_{0}$ is equivalent to showing that

$$
\left.\frac{\partial v}{\partial x_{n}}\right|_{x_{n}=0}<0
$$

We will adopt the approach from [Sc2]. Instead of the scalar curvature equation in [Sc2], we consider the system of elliptic partial differential equations (3.5). First recall from (3.3) that

$$
v(x)=(u \circ \psi(x))\left(\frac{2}{1+|x|^{2}}\right)^{\frac{n-4}{2}},
$$

where $u$ is a smooth function on $\Omega(\Gamma) \subset S^{n}$ and therefore is smooth at $\psi(\infty)=q \in$ $\partial B \subset \Omega(\Gamma)$, thus we have the following expansion

$$
v(x)=\frac{1}{|x|^{n-4}}\left(a_{0}+a_{i} \frac{x_{i}}{|x|^{2}}+a_{i j} \frac{x_{i} x_{j}}{|x|^{4}}+o\left(|x|^{-2}\right),\right.
$$

where $a_{0}>0$. Hence

$$
\begin{gathered}
\frac{\partial v}{\partial x_{n}}=-\frac{1}{|x|^{n-2}}\left((n-4) a_{0} x_{n}-a_{n}+(n-2) \frac{a_{i} x_{i}}{|x|^{2}} x_{n}+O\left(|x|^{-1}\right),\right. \\
w(x)=-\Delta v(x)=2(n+2) a_{0}|x|^{-n+2}+O\left(|x|^{-n}\right)
\end{gathered}
$$

and

$$
\frac{\partial w}{\partial x_{n}}=-2(n+2)(n-2) a_{0} x_{n}|x|^{-n}+O\left(|x|^{-n-1}\right) .
$$


Therefore there exist $C_{0}$ and $C_{1}$ such that

$$
\left\{\begin{array}{l}
\frac{\partial v}{\partial x_{n}}<0 \\
\frac{\partial w}{\partial x_{n}}<0
\end{array} \text { on the set }\left\{x \in R^{n}: x_{n} \geq C_{0}|x|^{-1} \&|x| \geq C_{1}\right\}\right.
$$

In the following we will use the moving plane method to conclude (3.11) from (3.16). For $\Lambda \in R$, let

$$
\Sigma_{\Lambda}=\left\{x \in R^{n}: x_{n}>\Lambda\right\} \text { and } S_{\Lambda}=\left\{x \in R^{n}: x_{n}=\Lambda\right\}
$$

We consider the reflection with respect to the hyperplane $x_{n}=\Lambda$

$$
x^{\lambda}=\left(x_{1}, x_{2}, \cdots 2 \Lambda-x_{n}\right)
$$

and define

$$
\left\{\begin{array}{c}
v^{\Lambda}(x)=v\left(x^{\Lambda}\right) \\
w^{\Lambda}(x)=w\left(x^{\Lambda}\right)
\end{array}\right.
$$

Then it is easily seen that

$$
\left\{\begin{array}{l}
-\Delta w^{\Lambda}(x)=\left(v^{\Lambda}(x)\right)^{\frac{n+4}{n-4}} \\
-\Delta v^{\Lambda}(x)=w^{\Lambda}(x)
\end{array} \quad \text { on } \hat{\Omega}^{\Lambda}\right.
$$

Similar to the proofs of Lemma 4.1 and Lemma 4.2 in [GNN] one knows that there exists $\Lambda_{0}$ such that, for each $\Lambda>\Lambda_{0}$,

$$
\left\{\begin{array}{c}
v^{\Lambda}(x)>v(x) \\
w^{\Lambda}(x)>w(x)
\end{array} \forall x \in \Sigma_{\Lambda} \backslash\left(\psi^{-1}(L(\Gamma))\right)^{\Lambda} .\right.
$$

Because of Lemma 3.1. Now let

$$
\Lambda^{*}=\inf \{\Lambda: \text { such that }(3.21) \text { holds }\} \text {. }
$$

Clearly $\Lambda^{*} \leq \Lambda_{0}$. Notice that, applying Hopf lemma for the elliptic system (cf. [Q])

$$
\left\{\begin{aligned}
-\Delta\left(w^{\Lambda}-w\right) & =\left(v^{\Lambda}\right)^{\frac{n+4}{n-4}}-v^{\frac{n+4}{n-4}} \\
-\Delta\left(v^{\Lambda}-v\right) & =w^{\Lambda}-w
\end{aligned} \quad \text { in } \Sigma_{\Lambda} \backslash\left(\psi^{-1}(L(\Gamma))\right)^{\Lambda} .\right.
$$


gives us

$$
0<\left.\frac{\partial\left(v^{\Lambda}-v\right)}{\partial x_{n}}\right|_{x_{n}=\Lambda}=-\left.2 \frac{\partial v}{\partial x_{n}}\right|_{x_{n}=\Lambda}, \forall \Lambda \geq \Lambda^{*}
$$

unless $v^{\Lambda} \equiv v$ which is possible only when the limit set $L(\Gamma)$ is empty, i.e. $(M, g)$ is conformally equivalent to $\left(S^{n}, g_{1}\right)$. Now, again similar to the proof of Lemma 4.4 in $[\mathrm{GNN}]$, we conclude that $\Lambda^{*}$ must be negative and the hyperplane $S_{\Lambda^{*}}$ should not be stopped before hitting the singular set $\psi^{-1}(L(\Gamma))$. Thus $\Lambda^{*} \leq 0$ and (3.11) holds.

\section{4. $L^{\infty}$ estimates and the proof of main theorem}

In this section we first use the blow-up method to prove the $L^{\infty}$ estimate for the conformal factor $u$, when $g_{u}=u^{\frac{4}{n-4}} g$ is a metric of positive scalar curvature and constant $Q$-curvature 1 . Then we use the positivity result Theorem 2.3 in the previous section to prove the $L^{\infty}$ estimate for $1 / u$. Higher order estimates follow from standard estimates for linear elliptic partial differential equations.

Theorem 4.1. Suppose that $(M, g)$ is a closed locally conformally flat manifold of dimension higher than 4 which is not conformally equivalent to $\left(S^{n}, g_{1}\right)$. Then there exists some constant $C>0$ such that, if $g_{u}=u^{\frac{4}{n-4}} \mathrm{~g}$ is a metric of positive scalar curvature and constant $Q$-curvature 1 , then

$$
\|u\|_{L^{\infty}(M)} \leq C
$$

Proof. Suppose otherwise we have a sequence of $u_{i}$ and a sequence of points $p_{i}$ on $M$ such that $\left(M, u_{i}^{\frac{4}{n-4}} g\right)$ has positive scalar curvature and $Q$-curvature equal to 1 and

$$
u_{i}\left(p_{i}\right)=\max _{p \in M} u_{i}(p) \rightarrow+\infty
$$

Let $s_{i} \in F \subset \Omega(\Gamma) \subset S^{n}$ such that $\phi\left(s_{i}\right)=p_{i}$, where $F$ is a fixed fundamental domain in $\Omega(\Gamma)$ for the Kleinian group $\Gamma$ and $\phi: \Omega(\Gamma) \rightarrow M$ is the covering map associated with the developing map. Without loss of generality, we may assume that $s_{i} \rightarrow s \in \bar{F} \subset \Omega(\Gamma)$. Then the universal covers $\left(\Omega(\Gamma), \tilde{u}_{i}^{\frac{4}{n-4}} g_{1}\right)$ for $\left(M, u_{i}^{\frac{4}{n-4}} g\right)$ satisfy the assumptions in Theorem 3.2 in the previous section. We choose a stereographic projection

$$
\psi: \hat{\Omega} \subset R^{n} \rightarrow \Omega(\Gamma) \subset S^{n}
$$


with respect to some appropriate point inside $\Omega(\Gamma)$ and work in the coordinates of $\left(\hat{\Omega}, \hat{u}_{i}^{\frac{4}{n-4}} g_{0}\right)$ for $\left(\Omega(\Gamma), \tilde{u}_{i}^{\frac{4}{n-4}} g_{1}\right)$. It is easily seen that there exists a $\delta>0$ such that $B_{\delta}\left(x_{i}\right) \subset \hat{\Omega}$, where $\phi\left(\psi\left(x_{i}\right)\right)=p_{i}$. Let

$$
v_{i}(x)=\frac{1}{u_{i}\left(p_{i}\right)} \hat{u}_{i}\left(x_{i}+\frac{x}{u_{i}^{\frac{2}{n-4}}\left(p_{i}\right)}\right), \forall x \in B_{u_{i}^{\frac{2}{n-4}}\left(p_{i}\right) \delta}(0) .
$$

One may verify that

$$
(-\Delta)^{2} v_{i}=v_{i}^{\frac{n+4}{n-4}}, \quad \text { on } B_{u_{i}^{\frac{2}{n-4}}\left(p_{i}\right) \delta}(0)
$$

and $0<v_{i} \leq 1$. Then standard elliptic estimates allow us to pick up a subsequence $v_{i_{k}}$ which converges to $v$ in the $C_{\text {loc }}^{4, \alpha}\left(R^{n}\right)$ topology, where

$$
(-\Delta)^{2} v=v^{\frac{n+4}{n-4}} \quad \text { on } R^{n}
$$

and $v \geq 0$. Because $-\Delta v \geq 0$ on $R^{n}$, which was proved in the proof of Theorem 3.1 in [WX], and $v(0)=1, v$ is actually positive. By a classification theorems in [WX], we know that $\left(R^{n}, v^{\frac{4}{n-4}} g_{0}\right)$ is the round sphere. Hence, a Euclidean ball $B_{K}(0)$ of sufficiently large radius is strictly geodesically concave in the round metric $v^{\frac{4}{n-4}} g_{0}$. Therefore the balls $B_{K}(0)$ are geodesically concave in the metrics $v_{i_{k}}^{\frac{4}{n-4}} g_{0}$ when $k$ is sufficiently large. That is to say the Euclidean ball $B_{u_{i_{k}}^{-1}\left(p_{i_{k}}\right) K}\left(x_{i_{k}}\right)$ is geodesically concave in the metric $\hat{u}_{i}^{\frac{4}{n-4}} g_{0}$, which is a contradiction with Theorem 3.2 in the previous section. Thus the proof is completed.

Now we state and prove our main theorem in this note.

Theorem 4.2. Let $(M, g)$ be a closed locally conformally flat manifold of dimension greater than 4 with positive Yamabe constant and Poincaré exponent less than $\frac{n-4}{2}$. Suppose that $(M, g)$ is not conformally equivalent to $\left(S^{n}, g_{1}\right)$ and that the Paneitz constant $P(M,[g])$ is positive. Then there exists $C_{k}>0$ for each $k \in N$ such that, if $g_{u}=u^{\frac{4}{n-4}} g$ is of positive scalar curvature and constant $Q$-curvature 1 , then

$$
\|u\|_{C^{k}(M)}+\left\|\frac{1}{u}\right\|_{C^{k}(M)} \leq C_{k}
$$

Proof. By the previous theorem and standard elliptic theory we only need to show that there is a uniform positive lower bound for the function $u$, if $g_{u}=u^{\frac{4}{n-4}} g$ is of 
positive scalar curvature and constant $Q$-curvature 1 . Suppose otherwise, there is a sequence of $u_{i}$ such that

$$
\min _{p \in M} u_{i}(p) \rightarrow 0
$$

Then, by the previous theorem and standard elliptic theory, we may take a subsequence $u_{i_{k}}$, which converges to $u \geq 0$ in $C^{4, \alpha}(M)$, where

$$
P[g] u=u^{\frac{n+4}{n-4}} \quad \text { on } M
$$

and $u(p)=0$ for some $p \in M$. But the existence of such $u$ contradicts with our Theorem 2.3 in Section 2 , unless $u \equiv 0$. If indeed $u \equiv 0$, then we would have

$$
\frac{\int_{M}(Q d v)\left[g_{u_{i_{k}}}\right]}{\left(\int_{M} d v\left[g_{u_{i_{k}}}\right]\right)^{\frac{n-4}{n}}}=\left(\int_{M} u_{i_{k}}^{\frac{2 n}{n-4}} d v_{g}\right)^{\frac{4}{n}} \rightarrow 0 \quad \text { as } k \rightarrow \infty
$$

which contradicts with the assumption that the Paneitz constant $P(M,[g])$ is positive. So the proof is finished.

\section{References:}

[Au] T. Aubin, The scalar curvature, differential geometry and relativity, (Cahen and Flato, eds.), Reidel, Dordrecht 1976.

[Br] Thomas Branson, Group representations arising from Lorentz conformal geometry, J. Func. Anal. 74 (1987), 199-291.

[CHY] S.-Y. A. Chang, F. Hang and P. Yang, On a class of locally conformally flat manifold, Preprint 2003.

[CQY2] S.-Y. A. Chang, J. Qing and P. Yang, On the finiteness of Kleinian groups in general dimensions, J. Reine Angew. Math., 571 (2004), 1-17.

[CY] S.-Y. A. Chang and P. Yang, Nonlinear differential equations in conformal geometry, Proceedings of ICM 2002.

[DHL] Z. Djadli, E. Hebey and M. Ledoux, Paneitz type operators and applications, Duke Math. J. 104(2000) no. 1, 129-169.

[DMA] Z. Djadli, A. Malchiodi and M. Ahmedou, Prescribing a fourth order conformal invariant on the standard sphere, Part II - blow-up analysis and applications, Preprint 2001. 
[GNN] B. Gidas, W.-M. Ni and L. Nirenberg, Symmetry and related properties via maximum principle, Comm. Math. Phys. 68 (1979), no. 3, 209-243.

[G] Maria Gonzalez, Singular sets of a class of locally conformally flat manifolds, Preprint 2004.

[HR] E. Hebey and E. Robert, Compactness and global estimates for the geometric equation in higher dimensions, E.R.A./A.M.S., Vol. 10, pp. 135-141, 2004.

[P] S. Paneitz, A quadratic conformally covariant differential operator for arbitrary pseudo-Riemannian manifolds, Preprint 1983.

[Q] Jie Qing, A Priori Estimates for Positive Solutions of Semi-linear Elliptic Systems. J. Partial Differential Equations, Vol.1, No.2, Series A(1988), pp 61-70.

[Sc1] Rick Schoen, Conformal deformation of a Riemmanian metric to constant scalar curvature, J. Diff. Geom. 6 (1984), 479-495.

[Sc2] Rick Schoen, On the number of constant scalar curvature metrics in a conformal class, Differential Geometry, 311-32o, Pitman Monogr. Surveys Pure Appl. Math., 52, Longman Sci. Tech., Harlow, 1991.

[SY] R. Schoen and S.T. Yau, Conformally flat manifolds, Kleinian groups, and scalar curvature, Invent. Math. 92 (1988), 47-71.

[Tr] N. Trudinger, Remarks concerning the conformal deformation of Riemannian structures on compact manifolds, Ann. Scuola Norm. Sup. Pisa Cl. Sci (3) 22 (1968), 265-274.

[WX] Juncheng Wei and Xingwang Xu, Classification of solutions of higher order conformally invariant equations, Math. Ann. 313 (1999), 207-228.

[Ya] H. Yamabe, On a deformation of Riemannian structures on compact manifolds, Osaka J. Math. 12 (1960), 21-37.

Jie Qing, Department of Mathematics, UC Santa Cruz, Santa Cruz, CA 95064

E-mail address: qing@ucsc.edu

David Raske, Department of Mathematics, UC Santa Cruz, Santa Cruz, CA 95064

E-mail address: gadfly@math.ucsc.edu 